\title{
Eletroestimulação para bexiga neurogênica em pacientes com lesão medular: Revisão sistemática
}

\author{
Electrical stimulation for neurogenic bladder in spinal \\ cord injury patients: Systematic review
}

MÔNICA SCHMOELLER Karina de Souza Tavares ${ }^{I}$ Erica Feio Carneiro Nunes ${ }^{\text {II }}$ Gustavo Fernando Sutter LATORRE $^{\text {III }}$

${ }^{\mathrm{I} U n i v e r s i d a d e ~ F e d e r a l ~ d o ~ P a r a n a ́ ~}$ (UFPR). Curitiba/PR - Brasil "Universidade do Estado do Pará (UEPA). Belém/PA - Brasil

"IIRede Perineo.net. Florianópolis/ SC - Brasil
Resumo Introdução: A lesão medular produz sequelas sobre a função urinária, e para estas existem poucas opções terapêuticas, entre as quais a eletroestimulação vem emergindo como técnica promissora. Objetivo: investigar as possibilidades de tratamento das sequelas sobre a função urinária de pacientes com lesão medular por eletroestimulação. Método: Revisão sistemática das bases de dados Pubmed, Lilacs e PEDro, buscando ensaios clínicos no período de 1980 a fevereiro de 2019, que trataram a bexiga neurogênica em indivíduos com lesão medular utilizando eletroestimulação. Resultados: Apenas cinco estudos preencheram os critérios de inclusão. Os parâmetros de eletroestimulação variaram enormemente, a boa parte dos estudos não alcançou significância estatística, mas de um modo geral apontaram uma tendência de que o tratamento melhorou os parâmetros urodinâmicos dos lesados medulares. Conclusões: Devido ao pequeno número de estudos, os diferentes métodos de eletroestimulação e variação de parâmetros e tempo de tratamento, não foi possível estabelecer consenso a respeito da eficácia das intervenções por eletroestimulação na função urinária de pacientes com lesão medular.

Palavras-chave: Eletroestimulação. Fisioterapia Pélvica. Lesão Medular. Bexiga.

Aвstract Background: Spinal cord injury produces sequelae on urinary function, and for these there are few therapeutic options, among which electrostimulation has emerged as a promising. Objective: To investigate the possibilities of treating sequelae on the urinary function of patients with spinal cord injury by electrostimulation. Method: Systematic review of the Pubmed, Lilacs and PEDro databases, seeking clinical trials from 1980 to February 2019, which treated the neurogenic bladder in individuals with spinal cord injury using electrostimulation. Results: Only five studies met the inclusion criteria. The electrostimulation parameters varied enormously, the majority of the studies did not reach statistical significance, but generally indicated a tendency that the treatment improved the urodynamic parameters of the spinal cord injured. Conclusion: Due to the small number of studies, the different methods of electrostimulation and variation of parameters and time of treatment, it was not possible to establish consensus regarding the effectiveness of electrostimulation interventions in the urinary function of patients with spinal cord injury.

Keywords: Electrostimulation. Pelvic Physiotherapy. Spinal CORD Injury. Bladder. 


\section{INTRODUÇÃO}

A bexiga é um órgão complexo devido à sua inervação e função, estando sujeita a alterações no nível de estruturas, de acordo com a Classificação Internacional de Funcionalidade (CIF), ${ }^{1}$ particularmente o de estruturas neurológicas. Essas alterações são responsáveis por comprometimentos no nível de função da CIF, causando incontinências ou retenções urinárias, estas, responsáveis por impactar negativamente sobre os níveis de atividades e participações sociais. Nesse contexto, as disfunções urinárias são um grande problema clínico e uma causa significativa de incapacidade em pacientes com lesão neurológica, como é o caso dos lesados medulares. ${ }^{2}$

Essa disfunção pode atingir qualquer parte do sistema voluntário e involuntário da função vesical, desde o centro miccional até os nervos terminais responsáveis pela bexiga (hipogástrico, pélvico e pudendo), passando pela medula, cadeia simpática, paravertebral e tronco cerebral. Ao nível de sinais e sintomas, a bexiga neurogênica (BN) pode aumentar a pressão intravesical, causar esvaziamento incompleto, inabilidade de iniciar ou de interromper a micção e incontinência, cada um desses problemas correlacionados diretamente a impactos sobre os níveis de estrutura e função. ${ }^{3,4}$

Desse modo, a disfunção neurogênica da bexiga é a complicação vesical mais comum nos serviços de reabilitação de pessoas com lesão medular, causando as chamadas Bexiga Hiperativa ou Bexiga Hipoativa, ${ }^{5}$ de acordo com a disfunção ser na fase do enchimento ou do esvaziamento vesical.

A BN Hiperativa é decorrente, normalmente, da interrupção dos feixes sensoriais e/ou motores da medula espinhal, que enviam impulsos entre a bexiga e o centro supraespinhal, comum nas lesões medulares acima do centro de micção. Já na BN Hipoativa a lesão ocorre no cone medular ou na cauda equina envolvendo o arco-reflexo, interrompendo as vias que transportam os impulsos sensoriais da bexiga para a medula espinhal, os impulsos motores da medula espinhal para o detrusor e para o esfíncter externo. ${ }^{6}$ A diferença principal, portanto, fica no nível medular ou altura da lesão.

Há falta de consenso na literatura a respeito de quais parâmetros utilizar na eletroestimulação para o tratamento de disfunções urinárias, ${ }^{7}$ tal fato se estende ao tratamento de pacientes específicos, como é o caso de lesados medulares. A investigação das possibilidades de tratamento das sequelas sobre a função urinária de pacientes com lesão medular por eletroestimulação é, por fim, o objetivo da presente revisão.

\section{Metodologia}

Trata-se de uma revisão sistemática realizada no mês de fevereiro de 2019. A busca ocorreu nas bases de dados Pubmed, LILACS e PEDro por meio dos descritores: eletroestimulação, bexiga neurogênica, fisioterapia e lesão medular e suas correspondentes em inglês: electral stimulation, neurogenic bladder, physical therapy, physiotherapy and spinal cord injury, utilizando os operadores boleanos and entre as palavras, e or entre physical therapy e physiotherapy.

Para a seleção dos estudos foram considerados elegíveis os ensaios clínicos, estudos retrospectivos com indivíduos e estudos pilotos em qualquer idioma e qualquer data. Foram incluídos estudos que trataram a bexiga neurogênica em indivíduos com lesão medular, utilizando qualquer tipo de estimulação elétrica, desde que aplicada de maneira transcutânea, com a intenção de 
desfecho para análise de melhora clínica. As intervenções foram consideradas inclusivas quando realizadas de forma isolada e sem envolvimento com outra técnica ou também quando fosse aplicada em terapia combinada e controlada. Foram incluídos estudos em pacientes com qualquer nível de lesão medular. Foram excluídos artigos duplicados nas bases de dados, estudos cujo tratamento não fosse de forma transcutânea e intravesical.

O processo de seleção do estudo envolveu a triagem dos títulos e leitura dos resumos, após o qual os artigos potencialmente relevantes foram obtidos no texto completo para uma análise mais aprofundada dos critérios de elegibilidade.

\section{Resultados}

As buscas brutas retornaram um total de 283 artigos. Após a remoção dos artigos duplicados e aplicação dos critérios de inclusão e exclusão, restaram apenas cinco estudos elegíveis. Os maiores motivos de inclusão foram abordar o efeito da eletroestimulação transcutânea em bexigas neurogênicas de pacientes com lesão medular, $o$ estudo estar disponível na íntegra, sem restrições de idiomas ou data, e os principais motivos de exclusão serem artigos duplicados nas bases de dados, tratando eletroestimulação por implante e/ou agulhamento (acupuntura), ou não apresentar o artigo integral (Figura 1).

Figura 1: Fluxogramas das buscas nas bases de dados.

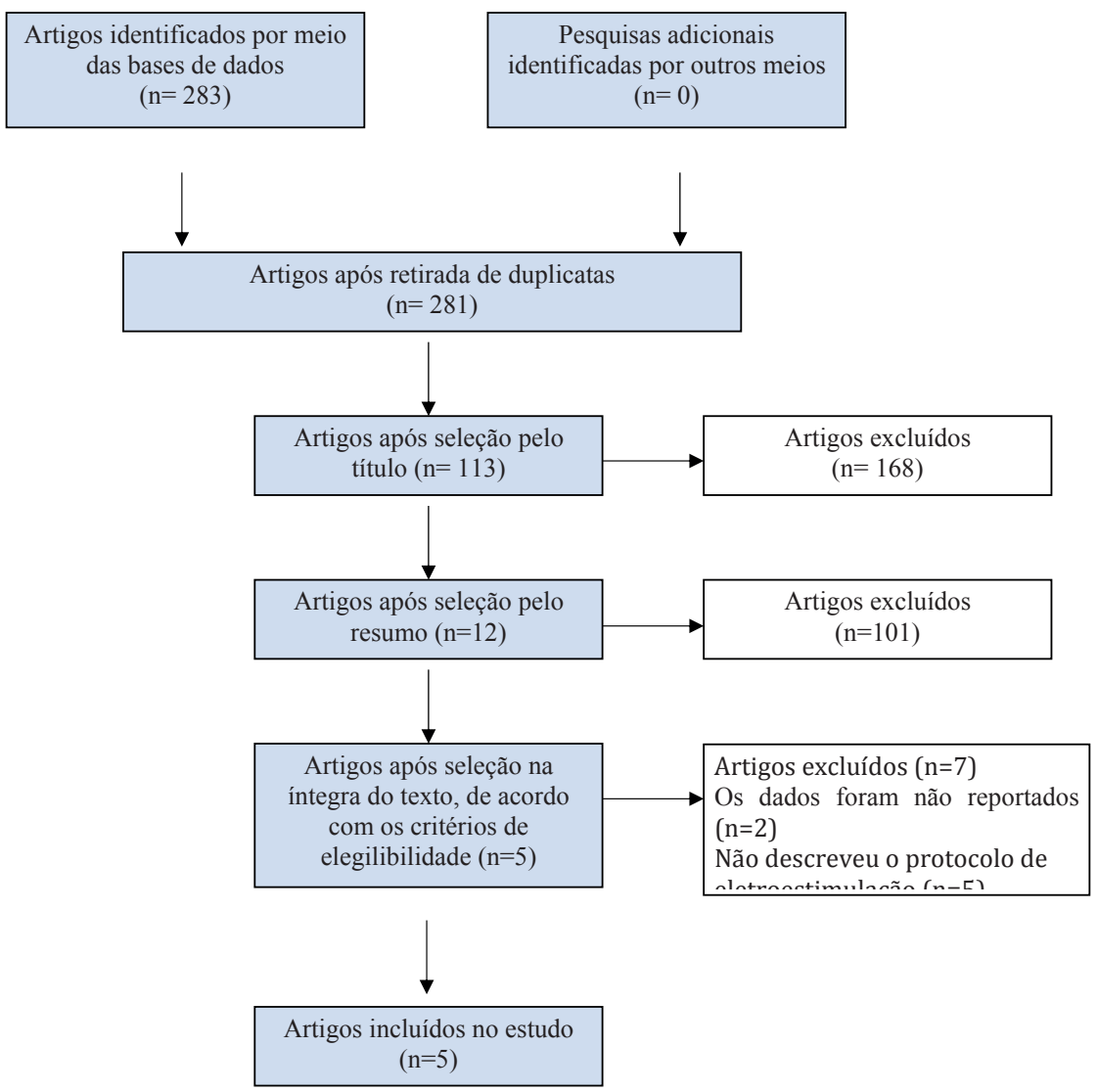


Dos cinco estudos incluídos, $\mathrm{um}^{8}$ abordou patologias associadas à BN, como Esclerose Múltipla, Doença de Parkinson e instabilidade detrusora idiopática. Dois estudos $^{8,10}$ utilizaram a Eletroestimulação intravesical (IVES), três estudos ${ }^{9,11,12}$ usaram a estimulação do nervo tibial posterior (PTNS) e apenas um ${ }^{11}$ empregou a estimulação do nervo dorsal do pênis (Tabela 1).

Quanto à dosagem, cada estudo apresentou um tempo diferente: um estudo com sessão única, ${ }^{9}$ um estudo com oito sessões, ${ }^{12}$ um estudo com 14 sessões, ${ }^{11}$ um estudo com 28 sessões ${ }^{10}$ e um com 50 sessões. ${ }^{8} \mathrm{O}$ tempo de cada sessão variou de 20 a 90 minutos.

Quanto aos instrumentos de medida, três estudos ${ }^{8,9,10}$ utilizaram o diário miccional, três ${ }^{8,9,11}$ usaram a cistometria, e dois d $^{8,10}$ utilizaram o estudo urodinâmico

O tipo de eletroestimulação utilizado foi a IVES ${ }^{8,10}$ e a PTNS. ${ }^{9,11,12}$ Os parâmetros da eletroestimulação variaram 10 a $100 \mathrm{hz}$, especificamente de 70 a $100 \mathrm{hz},{ }^{8,10} 10 \mathrm{hz},{ }^{9,10}$ e 20hz. ${ }^{11,12}$ A largura de pulso não foi informada em alguns estudos, ${ }^{8,10}$ mas nos estudos de Amarenco et al., ${ }^{9}$ Ojha et al. ${ }^{11}$ e Guoqing et al. ${ }^{12}$ foi utilizada a largura de pulso de 200 microssegundos.

\section{Discussão}

Nesta revisão sistemática, verificamos os efeitos da eletroestimulação transcutânea na função urinária de pacientes com lesão medular. Observamos que há indicadores de que a eletroestimulação em pacientes com lesão medular pode melhorar a função vesical, entretanto há inconsistências e falta de consenso entre os autores quanto ao tipo de eletroestimulação a ser usada, ao parâmetro da eletroestimulação e tempo terapêutico.

Madersbacher et al. ${ }^{8}$ e Lombardi et al. ${ }^{10}$ utilizaram a eletroestimulação intravesical, ambos apresentaram melhora dos parâmetros urodinâmico, porém tais melhoras não se mantiveram após um ano do fim do tratamento. Os autores defendem a estimulação diária, porém Madersbacher et al.$^{8}$ alcançaram seus resultados com média de 50 sessões contra apenas 28 de Lombardi et al. ${ }^{10}$. Os parâmetros eletroterapêuticos também variaram nos estudos: enquanto Madersbacher et al. ${ }^{8}$ defenderam uma frequência entre 70 a $100 \mathrm{~Hz}$, Lombardi et al. ${ }^{10}$ defenderam uma frequência abaixo dos $70 \mathrm{~Hz}$. Já Ojha et al., ${ }^{11}$ Guoqing et al. ${ }^{12}$ e Amarenco et al., ${ }^{9}$ defenderam uma largura de pulso $200 \mu$ s e frequência 10 a $20 \mathrm{~Hz}$. Ao que parece, qualquer frequência até $100 \mathrm{~Hz}$ pode ser terapêutica para esses casos, sendo necessários estudos investigando esta intrigante questão.

Em três estudos ${ }^{9,11,12}$ o uso da PTNS foi apontado como benéfico para os pacientes com lesão medular, porém nenhum dos três estudos alcançou diferença estatística significativa, de modo que a conclusão de que a corrente funciona para esses pacientes, até o presente, fica impossibilitada. Até agora a eletroestimulação por via tibial parece não surtir o efeito descrito na teoria e, por se tratar de uma técnica largamente utilizada por fisioterapeutas em geral, carece de estudos urgentes.

Atualmente, vem sendo debatida a PTNS, que pode ser feita com eletrodos de inserção (subcutâneos) ou eletrodos superficiais. ${ }^{13}$ Todavia, a literatura atual carece ainda de ao menos um ensaio randomizado comparando diretamente as duas modalidades, via agulha e via eletrodos de superfícies, uma vez que os 


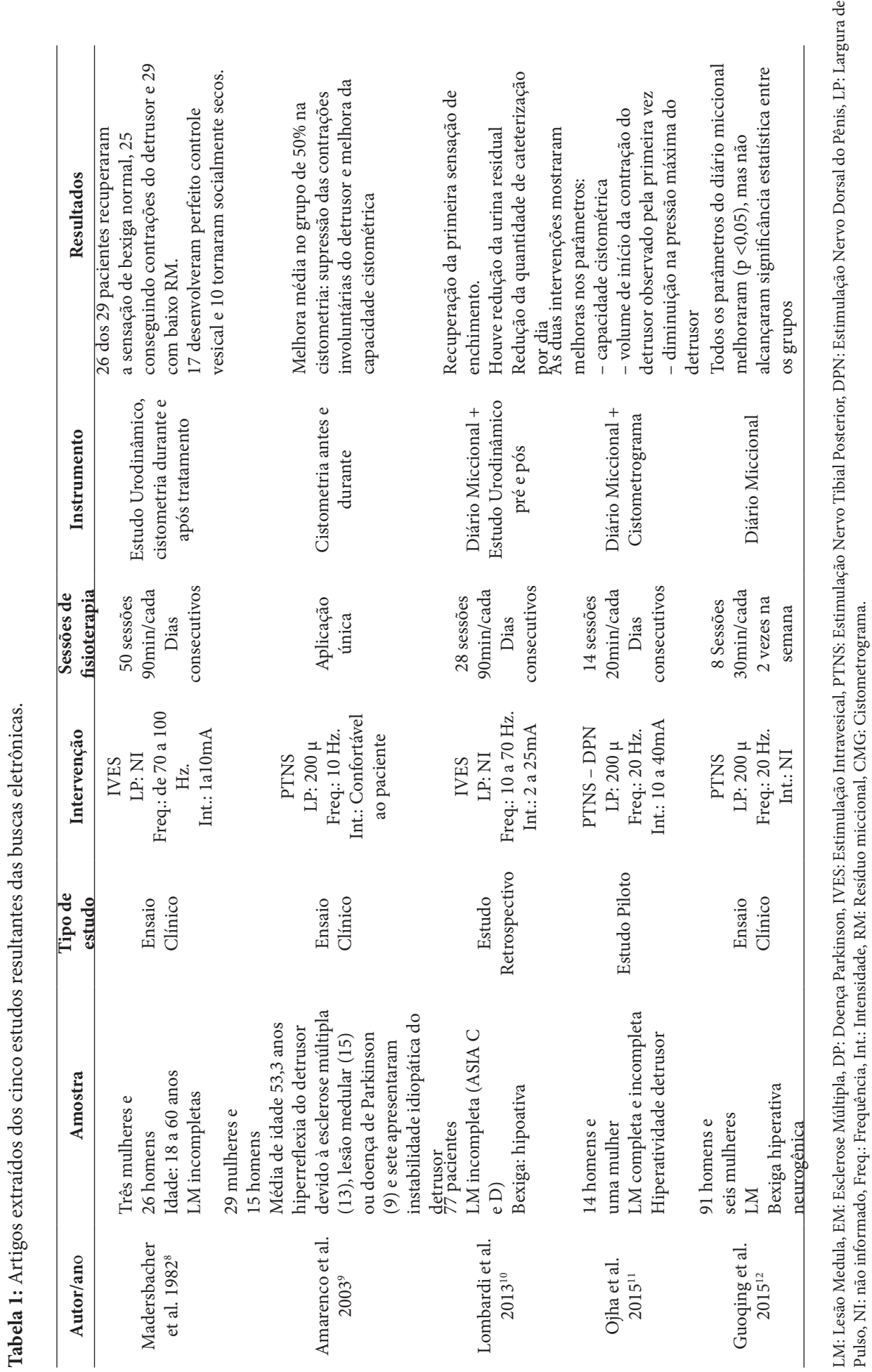


resultados satisfatórios descritos para a PTNS são com eletrodos de agulha. Até o presente, para a PTNS com eletrodos de superfície, existem apenas estudos pequenos e pouco significativos. No entanto, clinicamente a PTNS com eletrodos de superfície vem sendo utilizada, mesmo sem suficiente evidência de sua eficácia, pelo fato de ser mais acessível, e não invasiva, podendo vir a ser, caso comprovado, uma opção promissora para os casos de contraindicação ou intolerância à eletroestimulação intravaginal. ${ }^{13}$

Amarenco et al. ${ }^{9}$ apontaram que apenas uma sessão de eletroestimulação já apresentou resultados agudos nos parâmetros urodinâmicos. Ojha et al. ${ }^{11} \mathrm{e}$ Guoging et al. ${ }^{12}$ defenderam um número maior de sessões terapêuticas, sendo um com 14 e outro com oito sessões. Os resultados de Amarenco et al. ${ }^{9}$ degeneraram mais rapidamente com o tempo. Guoging et al. ${ }^{12}$ deixaram como suposição que um tempo maior de sessões poderia sim ser benéfico, sugerindo ainda um estimulador portátil para aplicação diária a longo tempo pelos próprios pacientes. Pode ser que a utilização domiciliar da eletroestimulação seja a resposta para a duração no longo prazo dos efeitos terapêuticos dessa modalidade em pacientes neurológicos, mas novos estudos se fazem necessários para comprovar essa hipótese.

O tratamento conservador de disfunções urinárias de origem neurogênica era realizado com drogas, mas a baixa eficácia e significativos efeitos colaterais favoreceram, nos últimos anos, a evolução da fisioterapia pélvica no manejo das sequelas funcionais desses pacientes. Assim, o treinamento dos músculos do assoalho pélvico, auxiliados ou não por biofeedback e/ou eletroestimulação, aliados a terapias comportamentais como o treinamento vesical, vêm conquistando cada vez mais espaço, passando a ser propostos na literatura como tratamento de primeira linha para as disfunções urinárias desses pacientes. ${ }^{14}$

O presente estudo teve como limitações o número reduzido de estudos primários. A eletroestimulação voltada para o tratamento da bexiga neurogênica, apesar de popular nos congressos, é cientificamente pouquíssima estudada. Em especial, quando a bexiga neurogênica é originada de uma lesão medular, o número de publicações é ainda mais escasso. A variação dos parâmetros usados para a eletroestimulação e os diferentes tempos de aplicação e utilização das intervenções, bem como, a heterogeneidade dos resultados avaliados comprometem as comparações dos efeitos encontrados entre os autores.

\section{CONSIDERAÇões FinaIS}

Devido ao pequeno número de estudos, os diferentes métodos de eletroestimulação e variação de parâmetros e tempo de tratamento, não foi possível estabelecer consenso a respeito da eficácia das intervenções por eletroestimulação na função urinária de pacientes com lesão medular. Todavia, ao que parece, a eletroestimulação tende a melhorar agudamente os parâmetros urodinâmicos de lesados medulares, e possivelmente a terapia domiciliar crônica possa garantir a manutenção dos efeitos no longo prazo. Por se tratar de técnica não invasiva e livre de efeitos colaterais, a eletroestimulação pode vir a ser considerada a primeira opção no tratamento das sequelas urinárias de lesados medulares, mas essa conclusão ainda necessita suporte em evidências mais robustas. 


\section{REFERÊNCIAS}

1. OMS - Organização Mundial de Saúde. Classificação Internacional de Funcionalidade, Incapacidade e Saúde - CIF. Versão Portuguesa, Lisboa, 2004, 238 p.

2. Amarenco G., Ismael SS, Chesnel C., Charlanes A., Breton FLE. Diagnosis and Clinical Evaluation of Neurogenic Bladder. Eur J Phys Rehabil Med. 2017; 53 (6): 975-980.

3. Rocha FET, Gomes CM. Urologia Fundamental. São Paulo: Planmark, 2010.

4. Martini AC, Forner S. Abordagem Multiprofissional em Lesão Medular: Saúde, Direito e /Organização de: Soraia Dornelles Schoeller. Florianópolis: Publicação do IFSC, 2016.

5. Tholl AD. O quotidiano e o ritmo de vida de pessoas com lesão medular e suas famílias: potências e limites na adesão à reabilitação para a Promoção da Saúde. 2015 250s. Tese (Doutorado em Filosofia na Enfermagem) - Curso de Pós-Graduação em Enfermagem, Universidade Federal de Santa Catarina, Florianópolis, 2015.

6. Stevens KA. Eliminação e continência urinária. In: Hoeman SP. Enfermagem de reabilitação: prevenção, intervenção e resultados esperados, 4. ed. Portugal: Lusodidacta, 2011.

7. Alves PGJM, Nunes FR, Guirro ECO. Comparison between two different neuromuscular electrical stimulation protocols for the treatment of female stress urinary incontinence: a randomized controlled trial. Rev. bras. Fisioter. 2011; 15 (5): 393-398.

8. Madersbacher H., Pauer W., Reiner E. Rehabilitation of micturition in patients with incomplete spinal cord lesions by transurethral electrostimulation of the bladder. Paraplegia. 1982, Aug.; 20 (4): 191-5

9. Amarenco G., Ismael SS, Even-Schneider A., Raibaut P., Demaille-Wlodyka S., Parratte B, Kerdraon J. Urodynamic effect of acute transcutaneous posterior tibial nerve stimulation in overactive bladder. J Urol. 2003; 169 (6): 2.210-5.

10. Lombardi G., et.al Eficácia clínica da eletroestimulação intravesical em pacientes com medula espinhal incompletos que sofrem de retenção neurogênica não-obstrutiva crônica: um estudo retrospectivo de centro único de 15 anos. Medula espinhal. 2013; 51: 232-237.

11. OJHAR, et.al. Neuromodulação por estimulação elétrica superficial de nervos periféricos para redução da hiperatividade detrusora em pacientes com lesão medular: um estudo piloto. J medula espinhal Med. 2015; 38 (2): 207-13.

12. Guoqing C., Liao L., Li Y. O possível papel da estimulação percutânea do nervo tibial usando eletrodos adesivos da superfície da pele em pacientes com hiperatividade neurogênica do detrusor secundária à lesão da medula espinhal. Int Urol Nephrol. 2015; 47: 451-5.

13. Franco M. de M., Souza F. de O., Vasconcelos ECLM de, Freitas MMS de, Ferreira CHJ. Avaliação da qualidade de vida e da perda urinária de mulheres com bexiga hiperativa tratadas com eletroestimulação transvaginal ou do nervo tibial. Fisioter. Pesqui. 2011; 18 (2): 145-150.

14. Bellette, P. O. Efeitos da eletroestimulação transcutânea do nervo tibial posterior sobre a sintomatologia e qualidade de vida em mulheres com bexiga hiperativa / Patrícia Odila Bellette. Campinas, 2007. 


\section{DADOS DOS AUTORES}

\section{Mônica Schmoeller}

Especialista em Fisioterapia Pélvica. Centro Catarinense de Reabilitação. Curitiba/PR Brasil. monifisioad@gmail.com

\section{Karina de Souza Tavares}

Especialista em Fisioterapia Pélvica. Clínica Vivafisio. Florianópolis/SC - Brasil. karina_ tvrs@hotmail.com

\section{Erica Feio Carneiro Nunes}

Doutora em Ciências da Reabilitação. Universidade do Estado do Pará. Belém/PA - Brasil. erica@perineo.net

\section{Gustavo Fernando Sutter LATORRE}

Mestre em fisioterapia. Gustavo Latorre Cursos. Florianópolis/SC - Brasil. gustavo@perineo.net

Submetido em: 29-7-2019

Aceito em: 12-4-2020 